\title{
Comparison of Age and Gender Differences of Tryptophan Metabolites in Patients of Major Monopolar and Bipolar Depression
}

\author{
Hiroi Tomioka ${ }^{1,2}$, Junichi Masuda ${ }^{3}$, Akikazu Takada4* ${ }^{*}$, Akira Iwanami ${ }^{1}$ \\ ${ }^{1}$ Department of Psychiatry, Showa University School of Medicine, Showa, Japan \\ ${ }^{2}$ Department of Psychiatry, Showa University Northern Yokohama Hospital, Showa, Japan \\ ${ }^{3}$ Global Application Development Center, Shimadzu Corporation, Kanagawa-ken, Japan \\ ${ }^{4}$ International Projects on Food and Health (NPO), Tokyo, Japan \\ Email: ^takadaa@mwd.biglobe.ne.jp
}

How to cite this paper: Tomioka, H., Masuda, J., Takada, A. and Iwanami, A. (2020) Comparison of Age and Gender Differences of Tryptophan Metabolites in Patients of Major Monopolar and Bipolar Depression. Food and Nutrition Sciences, 11, 172-185.

https://doi.org/10.4236/fns.2020.113013

Received: February 7, 2020

Accepted: March 2, 2020

Published: March 5, 2020

Copyright $\odot 2020$ by author(s) and Scientific Research Publishing Inc. This work is licensed under the Creative Commons Attribution International License (CC BY 4.0).

http://creativecommons.org/licenses/by/4.0/

\begin{abstract}
Background: No research has been done for the determination of plasma levels of tryptophan metabolites in patients of monopolar and bipolar depression. Methods: Ultra high-speed liquid chromatography/mass spectrometry has been used for the simultaneous determination of plasma levels of tryptophan metabolites in depressive patients. Results: No significant age and gender differences were shown in monopolar depressive patients and some differences were shown in bipolar patients. The administration of drugs such as antidepressants, antipsychotics, mood stabilizers do not seem to have affected the results. Conclusion: In patients of major monopolar depression age and gender differences of plasma levels of tryptophan metabolites disappear although significant differences are observed in healthy volunteers. Some differences of age and gender differences were shown between monopolar and bipolar depressive patients.
\end{abstract}

\section{Keywords}

Depression, Monopolar Depression, Bipolar Depression, Tryptophan, Serotonin, 5-Hydroxyindole Acetic Acid, Kynurenine, 3-Hydroxykynurenine, Kynurenic Acid, Anthranilic Acid, Xanthurenic Acid, Indole-3-Acetic Acid, SSRI (Selective Serotonin Reuptake Inhibitor), SNRI (Serotonin Norepinephrine Reuptake Inhibitor), Anxiolytic, Antipsychotic

\section{Introduction}

$22.8 \%$ of the global burden of diseases is due to psychiatric disorder [1]. Depres- 
sion is considered to be the leading cause of this disability. The number of people suffering from depression substantially increased since 1990, probably due to increase in aging people [2].

Antidepressants are widely used for the treatment of major depression. However, there has been a long lasting debate and concern about their efficacy and effectiveness. Generally, clinicians have a wide choice of individual drugs and they need evidence to make the best choice for patients.

Using a meta-analysis of data of the effectiveness of antidepressants, it has recently been shown that all antidepressants are more effective than placebo in adults with major depressive disorders [3].

Serotonin, one of important metabolites of TRP has been implicated to play roles of patho-etiology of depression, there debates about roles of serotonin.

Some people doubt roles of 5-HT in etiology of depression because 5-HT concentration was not decreased in the brains of depressive patients [4].

We have recently succeeded in the simultaneous measurements of all the TRP metabolites in plasma [5] [6] [7] [8]. Using this technology we measured all TRP metabolites in plasma of depressive patients.

We analyzed plasma levels of patients of major monopolar depression and preliminarily reported that plasma levels of 5-HT, but not 5-HIAA, were lower in depressive patients [6].

However, since there are significant gender and age differences in plasma levels of TRP metabolites in healthy volunteers, we decided to analyze differences of TRP metabolites in patients of major monopolar depression and bipolar depression.

\section{Methods}

1) Patients

Outpatients of depression were recruited in this study. Patients were diagnosed using the criteria of Diagnostic and Statistical Manual of Mental Disorders, the 5th edition (DSM-5, American Psychiatric Association 2013) [9]. Fasting blood samples were taken early in the morning. Their severity of depression was checked by CGI-S [10], SDS [11], and HDR-S [12]. The history of prescriptions of drugs such as antidepressants, anxyolitics, mood stabilizers and other drugs were asked.

Sample numbers are 55 (male; 15, and female 40, and average age; $45.4 \pm$ 11.9). The number of MDD is 36 and $\mathrm{BD}$ is 19 . Further characteristics of patients are described below. Outpatients who showed symptoms of depression were recruited in the studies at the Department of Psychiatry of Showa University Northern Yokohama Hospital. Patients were required to have had no lifetime history of psychosis, personality disorder, or drug or alcohol misuse. We also excluded patients who had organic problems of the brains. This study was carried out from 2016 to 2019. All patients gave their consent in a written form after receiving comprehensive information on the study protocol. 
2) Measurements of TRP metabolites.

Plasma factors were measured after plasma was separated from blood (3000 $\mathrm{rpm} / \mathrm{min}$. at $4^{\circ} \mathrm{C}$ ). Ethylenediaminetetraacetic acid (EDTA) was used as an anticoagulant.

The simultaneous measurements of TRP metabolites in plasma were performed by using an ultrahigh-speed liquid chromatography/spectrometry. Detailed methodology was described elsewhere [5] [6] [7] [8] [13]. TRP metabolic pathways are shown in Figure 1.

Figure 1 shows how each metabolite of TRP is located in the map.

\section{Statistics}

Statistical significance of the differences between groups was calculated according to one-way ANOVA. When ANOVA indicated a significant difference $(P<$ $0.05)$, the mean values of the treatment were compared using Tukey's least

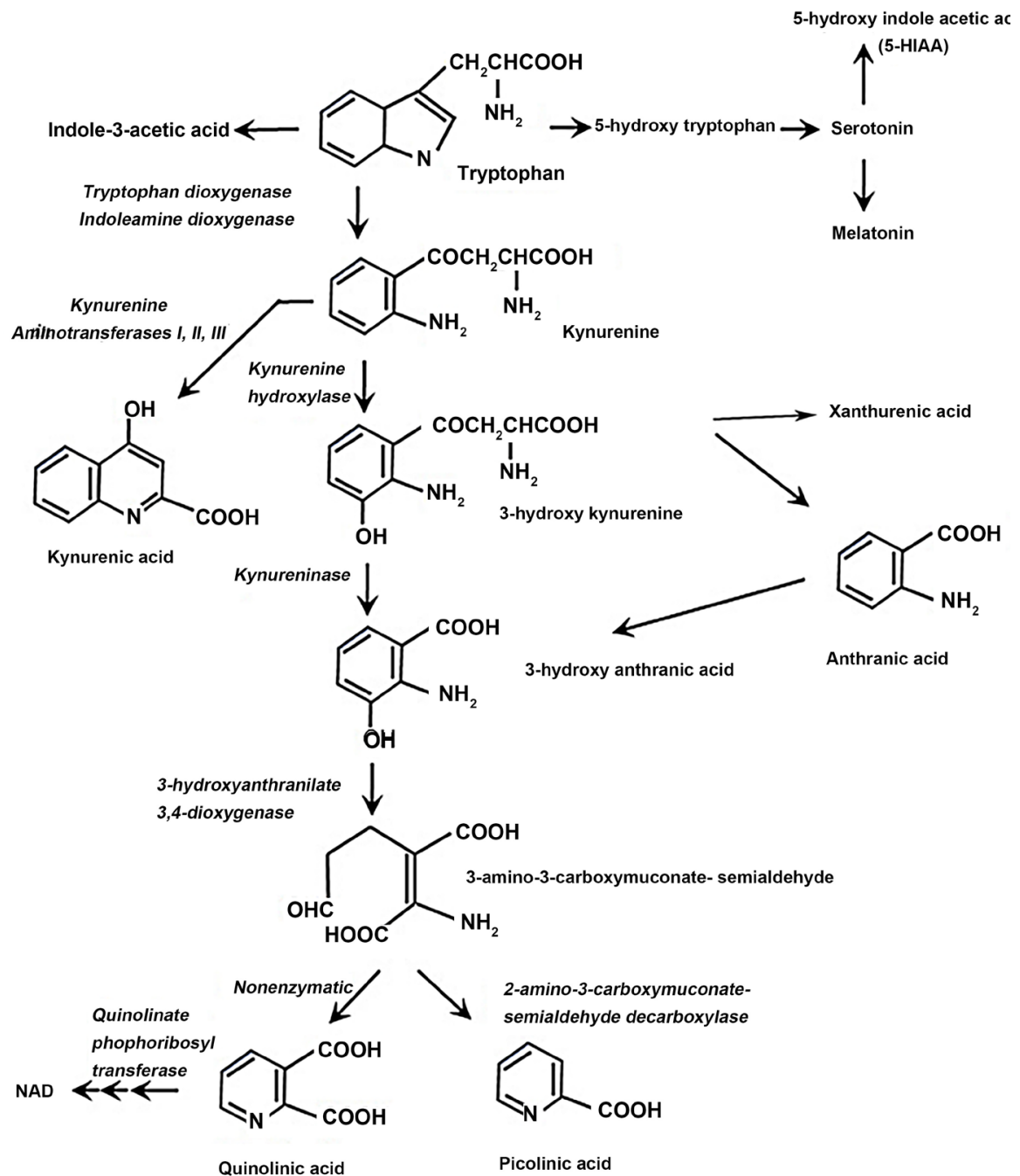

Figure 1. Metabolic pathway of TRP. There are three TRP metabolic pathways. Indole-3-acetic acid, serotonin and kynurenine pathways $\{7\}$. 
significant difference test at $P<0.05$. The statistical comparison between groups is analyzed by the two-way ANOVA. Mann-Whitney $U$ test was also used for evaluating statistical significance of the use of drugs.

\section{Results}

1) Tryptophan metabolites of patients with MDD.

Table 1 shows that by using one way ANOVA tests in patients of MDD only KYN levels of young men were higher than young women and old men. There are no significant difference in other metabolites among 4 groups.

Table 2 shows that KYN level of old men was higher that of other 3 groups and 5-HIAA level was higher that other 3 groups. The level of KYNA of old men was higher that that of young women.

Table 3 shows following results.

The results of two-way ANOVA were as follows:

${ }^{*} 1$ Age,

Diagnosis: F $(1,47)=0.298, p=0.588$; Sample name: $\mathrm{F}(3,47)=15.598, p<$ 0.01 ; and Diagnosis $\times$ Sample name interaction: $\mathrm{F}(3,47)=0.754, p=0.526$.

*2 Height,

Diagnosis $\times$ Sample name interaction: $\mathrm{F}(3,47)=2.991, p<0.05$.

*3 Weight,

Diagnosis: $\mathrm{F}(1,47)=3.358, p=0.074$; Sample name: $\mathrm{F}(3,47)=7.195, p<$ 0.01 ; and Diagnosis $\times$ Sample name interaction: $\mathrm{F}(3,47)=0.961, p=0.419$.

${ }^{\star} 4$ CGI-S,

Diagnosis $\times$ Sample name interaction: $\mathrm{F}(3,47)=3.585, p<0.05$.

*5 SDS,

Diagnosis: $\mathrm{F}(1,41)=0.003, p=0.955$; Sample name: $\mathrm{F}(3,41)=3.599, p<$ 0.05 ; and Diagnosis $\times$ Sample name interaction: $\mathrm{F}(3,41)=0.661, p=0.581$.

*6 HDR-S,

Diagnosis $\times$ Sample name interaction: $\mathrm{F}(3,47)=7.155, p<0.01$.

${ }^{*} 7$ Tryptophan (TRP),

Diagnosis: $\mathrm{F}(1,47)=0.070, \mathrm{p}=0.793$; Sample name: $\mathrm{F}(3,47)=4.131, p<$ 0.05 ; and Diagnosis $\times$ Sample name interaction: $\mathrm{F}(3,47)=1.962, p=0.133$.

*8 Kynurenine $(\mathrm{KYN})$,

Diagnosis $\times$ Sample name interaction: $\mathrm{F}(3,47)=6.538, p<0.01$.

*9 5-Hydroxyindoleacetic acid (5-HIAA),

Diagnosis $\times$ Sample name interaction: $\mathrm{F}(3,47)=3.129, p<0.05$.

${ }^{\star} 10$ Kynurenic acid,

Diagnosis $\times$ Sample name interaction: $\mathrm{F}(3,47)=7.405, p<0.01$.

*11 Xanthurenic acid,

Diagnosis $\times$ Sample name interaction: $\mathrm{F}(3,47)=3.222, p<0.005$.

The ratios of products per substrate (TRP) indicate the rates of the production of the products. Table 4 shows that there was no significant difference in the rates of the production of the products from TRP in patients of MDD and BD. 
Table 1. Tryptophan metabolites of patients with MDD.

\begin{tabular}{|c|c|c|c|c|c|}
\hline \multirow{2}{*}{$\begin{array}{c}\text { Diagnosis } \\
\text { Sample name }\end{array}$} & \multicolumn{4}{|c|}{$\begin{array}{l}\text { MDD } \\
\mathrm{n}=38\end{array}$} & \multirow[b]{2}{*}{$\begin{array}{l}\text { significant } \\
\text { differences }\end{array}$} \\
\hline & $\begin{array}{c}\text { young men } \\
\mathrm{n}=7 \\
\mathrm{a}\end{array}$ & $\begin{array}{c}\text { young women } \\
\mathrm{n}=18 \\
\mathrm{~b}\end{array}$ & $\begin{array}{c}\text { old men } \\
\mathrm{n}=3 \\
\mathrm{c}\end{array}$ & $\begin{array}{c}\text { old women } \\
\mathrm{n}=10 \\
\mathrm{~d}\end{array}$ & \\
\hline Age (years) & $36.00 \pm 9.256$ & $40.00 \pm 7.004$ & $60.00 \pm 14.73$ & $59.40 \pm 7.306$ & $\begin{array}{l}\text { a vs c: } p<0.01 \\
\text { a vs d: } p<0.01 \\
\text { b vs c: } p<0.01 \\
\text { b vs d: } p<0.01 \\
\text { b vs d: } p<0.01\end{array}$ \\
\hline Height (m) & $1.72 \pm 0.07$ & $1.59 \pm 0.06$ & $1.64 \pm 0.10$ & $1.59 \pm 0.05$ & $\begin{array}{l}\text { a vs b: } p<0.01 \\
\text { a vs } \mathrm{d}: p<0.01\end{array}$ \\
\hline Weight $(\mathrm{kg})$ & $74.41 \pm 12.86$ & $55.92 \pm 7.670$ & $58.95 \pm 16.05$ & $54.52 \pm 8.389$ & $\begin{array}{l}\text { a vs b: } p<0.01 \\
\text { a vs d: } p<0.01\end{array}$ \\
\hline BMI & $24.97 \pm 3.998$ & $22.31 \pm 4.051$ & $22.51 \pm 2.772$ & $21.58 \pm 2.982$ & - \\
\hline CGI-S & $4.429 \pm 0.535$ & $3.667 \pm 0.907$ & $4.667 \pm 0.577$ & $3.700 \pm 0.483$ & - \\
\hline SDS & $62.43 \pm 6.754$ & $45.00 \pm 13.31$ & $58.00 \pm 1.414$ & $52.22 \pm 6.399$ & a vs b: $p<0.01$ \\
\hline HDR-S & $21.29 \pm 3.817$ & $16.94 \pm 5.150$ & $29.67 \pm 5.033$ & $18.30 \pm 4.398$ & $\begin{array}{l}\text { b vs } \mathrm{c}: p<0.01 \\
\text { c vs d: } p<0.01\end{array}$ \\
\hline ID2: Triptophan $(\mu \mathrm{M})$ & $64.09 \pm 13.77$ & $58.48 \pm 10.40$ & $59.11 \pm 2.026$ & $51.24 \pm 11.01$ & - \\
\hline ID3: L-5-Hydroxytryptophan (nM) & $11.26 \pm 3.447$ & $9.191 \pm 2.942$ & 1.1800000 & $8.106 \pm 6.660$ & - \\
\hline ID4: Serotonin (nM) & $325.6 \pm 351.4$ & $267.6 \pm 204.4$ & $120.3 \pm 96.01$ & $160.2 \pm 145.2$ & - \\
\hline ID5: Kynurenine (nM) & $2506.5 \pm 1042.9$ & $1653.0 \pm 510.16$ & $1014.2 \pm 418.25$ & $1666.4 \pm 534.60$ & $\begin{array}{l}\text { a vs b: } p<0.05 \\
\text { a vs c: } p<0.05\end{array}$ \\
\hline ID6: 5-Hydroxytryptophol (nM) & - & 10.50 & 25.11 & 2.20 & - \\
\hline ID7: Tryptophol (nM) & 2.2950000 & $12.00 \pm 18.10$ & - & $11.99 \pm 11.18$ & - \\
\hline ID8: 5-Hydroxyindoleacetic acid (nM) & $28.04 \pm 22.07$ & $35.00 \pm 28.99$ & $22.82 \pm 28.11$ & $48.68 \pm 58.22$ & - \\
\hline ID9 :Indole-3-acetic acid (nM) & $2631.3 \pm 1666.0$ & $2068.4 \pm 1525.8$ & $583.0 \pm 266.4$ & $2412.5 \pm 1026.1$ & - \\
\hline ID10: Anthranilic acid (nM) & 5.61 & $14.29 \pm 11.76$ & - & $13.89 \pm 21.11$ & - \\
\hline ID11: Kynurenic acid (nM) & $67.07 \pm 39.75$ & $32.09 \pm 15.37$ & $26.47 \pm 12.76$ & $36.57 \pm 14.38$ & - \\
\hline ID12: Quinaldic acid (nM) & $10.14 \pm 7.55$ & $7.46 \pm 3.27$ & - & $7.81 \pm 3.96$ & - \\
\hline ID13: 3-Indolebutyric acid (nM) & $18.93 \pm 12.54$ & $18.45 \pm 11.93$ & $2.92 \pm 4.04$ & $25.62 \pm 20.27$ & - \\
\hline ID14: 3-Hydroxykynurenine (nM) & $19.03 \pm 14.17$ & $20.55 \pm 11.06$ & $29.96 \pm 8.09$ & $13.98 \pm 5.98$ & - \\
\hline ID15: 3-hydroxyanthranilic acid (nM) & $27.37 \pm 11.08$ & $28.12 \pm 21.21$ & - & $26.85 \pm 14.18$ & - \\
\hline ID16: Xanthurenic acid (nM) & $19.50 \pm 11.06$ & $10.12 \pm 6.17$ & $11.88 \pm 1.91$ & $13.05 \pm 5.79$ & - \\
\hline ID17: Melatonin (nM) & 0.17 & $6.61 \pm 8.26$ & - & $25.85 \pm 36.24$ & - \\
\hline ID19: Quinolinic acid (positive) (nM) & $776.1 \pm 1091.6$ & $404.6 \pm 277.7$ & $168.5 \pm 5.9$ & $334.1 \pm 303.9$ & - \\
\hline
\end{tabular}

One-way ANOVA was used for evaluating statical significance. A, b, c, d indicate values of young and old men and women.

Table 4 shows that 5 -HIAA/TRP ration was higher in BD patients than MMD patients.

Table 5 shows that the presence of old men resulted in increase in 5-HIAA/TRP ratio.

2-2; Influences of drug administrations on substrate product ratios. 
Table 2. Tryptophan metabolites of patients with BD.

\begin{tabular}{|c|c|c|c|c|c|}
\hline \multirow{2}{*}{$\begin{array}{c}\text { Diagnosis } \\
\text { Sample name }\end{array}$} & \multicolumn{4}{|c|}{$\begin{array}{c}\mathrm{BD} \\
\mathrm{n}=17\end{array}$} & \multirow[b]{2}{*}{$\begin{array}{l}\text { significant } \\
\text { differences }\end{array}$} \\
\hline & $\begin{array}{c}\text { young men } \\
\mathrm{n}=2 \\
\mathrm{a}\end{array}$ & $\begin{array}{c}\text { young women } \\
\mathrm{n}=10 \\
\mathrm{~b}\end{array}$ & $\begin{array}{c}\text { old men } \\
\mathrm{n}=3 \\
\mathrm{c}\end{array}$ & $\begin{array}{c}\text { old women } \\
\mathrm{n}=2 \\
\mathrm{~d}\end{array}$ & \\
\hline Age (years) & $40.50 \pm 0.707$ & $39.40 \pm 8.140$ & $58.33 \pm 4.619$ & $51.00 \pm 0.000$ & $\begin{array}{l}\mathrm{b} \text { vs } \mathrm{c}: p<0.01 \\
\mathrm{~b} \text { vs } \mathrm{d}: p<0.01\end{array}$ \\
\hline Height (m) & $1.63 \pm 0.00$ & $1.58 \pm 0.04$ & $1.73 \pm 0.04$ & $1.56 \pm 0.01$ & $\begin{array}{l}\text { b vs c: } p<0.01 \\
\text { c vs d: } p<0.01\end{array}$ \\
\hline Weight $(\mathrm{kg})$ & $73.00 \pm 9.899$ & $59.34 \pm 2.215$ & $74.17 \pm 7.522$ & $60.30 \pm 3.677$ & $\begin{array}{l}\text { b vs c: } p<0.01 \\
\text { c vs d: } p<0.05\end{array}$ \\
\hline BMI & $27.48 \pm 3.726$ & $23.69 \pm 1.371$ & $25.02 \pm 3.572$ & $24.77 \pm 1.062$ & - \\
\hline CGI-S & $4.000 \pm 0.000$ & $4.100 \pm 0.738$ & $3.000 \pm 1.000$ & $4.000 \pm 0.000$ & - \\
\hline SDS & $63.00 \pm 4.243$ & $51.56 \pm 7.986$ & $44.33 \pm 8.387$ & $55.00 \pm 7.071$ & - \\
\hline HDR-S & $18.00 \pm 0.000$ & $17.70 \pm 5.012$ & $11.00 \pm 2.000$ & $15.00 \pm 2.828$ & - \\
\hline ID2: Triptophan $(\mu \mathrm{M})$ & $57.71 \pm 2.006$ & $61.68 \pm 16.96$ & $78.50 \pm 10.30$ & $39.66 \pm 11.91$ & - \\
\hline ID3: L-5-Hydroxytryptophan (nM) & - & $7.598 \pm 0.886$ & 1.2260000 & - & - \\
\hline ID4: Serotonin (nM) & $302.0 \pm 45.28$ & $459.7 \pm 402.5$ & $250.3 \pm 184.6$ & $200.5 \pm 128.0$ & - \\
\hline ID5: Kynurenine (nM) & $1119.0 \pm 161.08$ & $1389.8 \pm 342.36$ & $2426.2 \pm 628.86$ & $914.89 \pm 73.22$ & $\begin{array}{l}\text { a vs c: } p<0.05 \\
\text { b vs c: } p<0.01 \\
\text { c vs d: } p<0.01\end{array}$ \\
\hline ID6: 5-Hydroxytryptophol (nM) & - & - & - & - & - \\
\hline ID7: Tryptophol (nM) & - & - & - & - & - \\
\hline ID8: 5-Hydroxyindoleacetic acid (nM) & $10.62 \pm 6.362$ & $17.01 \pm 13.09$ & $29.83 \pm 7.324$ & $116.83 \pm 27.63$ & $\begin{array}{l}\text { a vs d: } p<0.01 \\
\text { b vs d: } p<0.01 \\
\text { c vs d: } p<0.01\end{array}$ \\
\hline ID9 :Indole-3-acetic acid (nM) & $852.8 \pm 418.1$ & $1500.7 \pm 959.3$ & $835.1 \pm 217.5$ & $1184.4 \pm 1195.2$ & - \\
\hline ID10: Anthranilic acid (nM) & - & 11.59 & - & - & \\
\hline ID11: Kynurenic acid (nM) & $24.87 \pm 14.39$ & $41.91 \pm 25.76$ & $113.43 \pm 60.29$ & $24.55 \pm 18.43$ & b vs c: $p<0.05$ \\
\hline ID12: Quinaldic acid (nM) & - & $9.97 \pm 4.84$ & $1.99 \pm 0.86$ & - & - \\
\hline ID13: 3-Indolebutyric acid (nM) & $4.39 \pm 0.49$ & $19.94 \pm 18.23$ & $2.95 \pm 0.00$ & $5.72 \pm 1.80$ & - \\
\hline ID14: 3-Hydroxykynurenine (nM) & $28.66 \pm 0.35$ & $29.00 \pm 16.64$ & $25.91 \pm 9.04$ & $10.21 \pm 13.06$ & - \\
\hline ID15: 3-hydroxyanthranilic acid (nM) & $0.52 \pm 0.00$ & $19.91 \pm 21.48$ & $18.15 \pm 14.70$ & - & - \\
\hline ID16: Xanthurenic acid (nM) & $7.65 \pm 7.65$ & $16.48 \pm 13.72$ & $30.84 \pm 13.55$ & 9.75 & - \\
\hline ID17: Melatonin (nM) & - & - & - & - & - \\
\hline ID19: Quinolinic acid (positive) (nM) & $168.2 \pm 2.8$ & $259.2 \pm 200.1$ & $227.7 \pm 96.9$ & $134.3 \pm 13.3$ & - \\
\hline
\end{tabular}

One-way ANOVA was used for evaluating statistical significance. A,b,c,d indicate values of young and old men and women.

Table 6 shows that using $\mathrm{U}$ test, KYN/TRP ratio was higher in patients who took SSRI/SNRI.

\section{Discussion}

TRP is an essential amino acid needed not only for the protein synthesis but its metabolites have important functions in many physiological and pathological 
Table 3. Comparison of tryptophan metabolites between MDD and BD.

\begin{tabular}{|c|c|c|c|c|c|c|c|c|c|}
\hline \multirow{2}{*}{$\begin{array}{c}\text { Diagnosis } \\
\text { Sample name }\end{array}$} & \multicolumn{4}{|c|}{$\begin{array}{l}\mathrm{MDD} \\
\mathrm{n}=38\end{array}$} & \multicolumn{4}{|c|}{$\begin{array}{c}\mathrm{BD} \\
\mathrm{n}=17\end{array}$} & \multirow{2}{*}{$\begin{array}{l}\text { significant } \\
\text { differences }\end{array}$} \\
\hline & $\begin{array}{c}\text { young men } \\
\mathrm{n}=7 \\
\mathrm{a}\end{array}$ & $\begin{array}{c}\text { young women } \\
\mathrm{n}=18 \\
\mathrm{~b}\end{array}$ & $\begin{array}{c}\text { old men } \\
\mathrm{n}=3 \\
\mathrm{c}\end{array}$ & $\begin{array}{c}\text { old women } \\
\mathrm{n}=10 \\
\mathrm{~d}\end{array}$ & $\begin{array}{c}\text { young men } \\
\mathrm{n}=2 \\
\mathrm{a}\end{array}$ & $\begin{array}{c}\text { young women } \\
\mathrm{n}=10 \\
\mathrm{~b}\end{array}$ & $\begin{array}{c}\text { old men } \\
\mathrm{n}=3 \\
\mathrm{c}\end{array}$ & $\begin{array}{c}\text { old women } \\
\mathrm{n}=2 \\
\mathrm{~d}\end{array}$ & \\
\hline Age (years) & $36.00 \pm 9.256$ & $40.00 \pm 7.004$ & $60.00 \pm 14.73$ & $59.40 \pm 7.306$ & $40.50 \pm 0.707$ & $39.40 \pm 8.140$ & $58.33 \pm 4.619$ & $51.00 \pm 0.000$ & *1 \\
\hline Height (m) & $1.72 \pm 0.07$ & $1.59 \pm 0.06$ & $1.64 \pm 0.10$ & $1.59 \pm 0.05$ & $1.63 \pm 0.00$ & $1.58 \pm 0.04$ & $1.73 \pm 0.04$ & $1.56 \pm 0.01$ & $\star 2$ \\
\hline Weight (kg) & $74.41 \pm 12.86$ & $55.92 \pm 7.670$ & $58.95 \pm 16.05$ & $54.52 \pm 8.389$ & $73.00 \pm 9.899$ & $59.34 \pm 2.215$ & $74.17 \pm 7.522$ & $60.30 \pm 3.677$ & *3 \\
\hline BMI & $24.97 \pm 3.998$ & $22.31 \pm 4.051$ & $22.51 \pm 2.772$ & $21.58 \pm 2.982$ & $27.48 \pm 3.726$ & $23.69 \pm 1.371$ & $25.02 \pm 3.572$ & $24.77 \pm 1.062$ & - \\
\hline CGI-S & $4.429 \pm 0.535$ & $3.667 \pm 0.907$ & $4.667 \pm 0.577$ & $3.700 \pm 0.483$ & $4.000 \pm 0.000$ & $4.100 \pm 0.738$ & $3.000 \pm 1.000$ & $4.000 \pm 0.000$ & ${ }^{*} 4$ \\
\hline SDS & $62.43 \pm 6.754$ & $45.00 \pm 13.31$ & $58.00 \pm 1.414$ & $52.22 \pm 6.399$ & $63.00 \pm 4.243$ & $51.56 \pm 7.986$ & $44.33 \pm 8.387$ & $55.00 \pm 7.071$ & *5 \\
\hline HDR-S & $21.29 \pm 3.817$ & $16.94 \pm 5.150$ & $29.67 \pm 5.033$ & $18.30 \pm 4.398$ & $18.00 \pm 0.000$ & $17.70 \pm 5.012$ & $11.00 \pm 2.000$ & $15.00 \pm 2.828$ & *6 \\
\hline ID2: Tryptophan $(\mu \mathrm{M})$ & $64.09 \pm 13.77$ & $58.48 \pm 10.40$ & $59.11 \pm 2.026$ & $51.24 \pm 11.01$ & $57.71 \pm 2.006$ & $61.68 \pm 16.96$ & $78.50 \pm 10.30$ & $39.66 \pm 11.91$ & $\star^{* 7}$ \\
\hline ID3: L-5-Hydroxytryptophan (nM) & $11.26 \pm 3.447$ & $9.191 \pm 2.942$ & 1.1800000 & $8.106 \pm 6.660$ & - & $7.598 \pm 0.886$ & 1.2260000 & - & - \\
\hline ID4: Serotonin (nM) & $325.6 \pm 351.4$ & $267.6 \pm 204.4$ & $120.3 \pm 96.01$ & $160.2 \pm 145.2$ & $302.0 \pm 45.28$ & $459.7 \pm 402.5$ & $250.3 \pm 184.6$ & $200.5 \pm 128.0$ & - \\
\hline ID5: Kynurenine (nM) & $2506.5 \pm 1042.9$ & $1653.0 \pm 510.16$ & $1014.2 \pm 418.25$ & $1666.4 \pm 534.60$ & $1119.0 \pm 161.08$ & $1389.8 \pm 342.36$ & $2426.2 \pm 628.86$ & $914.89 \pm 73.22$ & $* 8$ \\
\hline ID6: 5-Hydroxytryptophol (nM) & - & 10.50 & 25.11 & 2.20 & - & - & - & - & - \\
\hline ID7: Tryptophol (nM) & 2.2950000 & $12.00 \pm 18.10$ & - & $11.99 \pm 11.18$ & - & - & - & - & - \\
\hline $\begin{array}{l}\text { ID8: 5-Hydroxyindoleacetic } \\
\text { acid (nM) }\end{array}$ & $28.04 \pm 22.07$ & $35.00 \pm 28.99$ & $22.82 \pm 28.11$ & $48.68 \pm 58.22$ & $10.62 \pm 6.362$ & $17.01 \pm 13.09$ & $29.83 \pm 7.324$ & $116.83 \pm 27.63$ & *9 \\
\hline ID9 :Indole-3-acetic acid (nM) & $2631.3 \pm 1666.0$ & $2068.4 \pm 1525.8$ & $583.0 \pm 266.4$ & $2412.5 \pm 1026.1$ & $852.8 \pm 418.1$ & $1500.7 \pm 959.3$ & $835.1 \pm 217.5$ & $1184.4 \pm 1195.2$ & - \\
\hline ID10: Anthranilic acid (nM) & 5.61 & $14.29 \pm 11.76$ & - & $13.89 \pm 21.11$ & - & 11.59 & - & - & - \\
\hline ID12: Quinaldic acid (nM) & $10.14 \pm 7.55$ & $7.46 \pm 3.27$ & - & $7.81 \pm 3.96$ & - & $9.97 \pm 4.84$ & $1.99 \pm 0.86$ & - & - \\
\hline ID13: 3-Indolebutyric acid (nM) & $18.93 \pm 12.54$ & $18.45 \pm 11.93$ & $2.92 \pm 4.04$ & $25.62 \pm 20.27$ & $4.39 \pm 0.49$ & $19.94 \pm 18.23$ & $2.95 \pm 0.00$ & $5.72 \pm 1.80$ & - \\
\hline ID14: 3-Hydroxykynurenine (nM) & $19.03 \pm 14.17$ & $20.55 \pm 11.06$ & $29.96 \pm 8.09$ & $13.98 \pm 5.98$ & $28.66 \pm 0.35$ & $29.00 \pm 16.64$ & $25.91 \pm 9.04$ & $10.21 \pm 13.06$ & - \\
\hline $\begin{array}{l}\text { ID15: 3-hydroxyanthranilic } \\
\text { acid (nM) }\end{array}$ & $27.37 \pm 11.08$ & $28.12 \pm 21.21$ & - & $26.85 \pm 14.18$ & $0.52 \pm 0.00$ & $19.91 \pm 21.48$ & $18.15 \pm 14.70$ & - & - \\
\hline ID16: Xanthurenic acid (nM) & $19.50 \pm 11.06$ & $10.12 \pm 6.17$ & $11.88 \pm 1.91$ & $13.05 \pm 5.79$ & $7.65 \pm 7.65$ & $16.48 \pm 13.72$ & $30.84 \pm 13.55$ & 9.75 & ${ }^{\star} 11$ \\
\hline ID17: Melatonin (nM) & 0.17 & $6.61 \pm 8.26$ & - & $25.85 \pm 36.24$ & - & - & - & - & - \\
\hline $\begin{array}{l}\text { ID19: Quinolinic acid } \\
\text { (positive) (nM) }\end{array}$ & $776.1 \pm 1091.6$ & $404.6 \pm 277.7$ & $168.5 \pm 5.9$ & $334.1 \pm 303.9$ & $168.2 \pm 2.8$ & $259.2 \pm 200.1$ & $227.7 \pm 96.9$ & $134.3 \pm 13.3$ & - \\
\hline
\end{tabular}

Two-way ANOVA with diagnosis (MDD and BD) and sample name (young and old men and women) was used for evaluating statistical significance.

processes [14]. Humans do not synthesize TRP, which must be taken from diet. The required daily dose is considered to be $3.5 \mathrm{mg}$ per $\mathrm{kg}$ of weight [15]. Less than $1 \%$ of ingested TRP is used for protein synthesis and the majority of TRP is metabolized along three known pathways, Indole-3-acetic acid (IAA), 5-HT and KYN pathways. The majority of TRP is enters from the gut to portal circulation and metabolized in the liver. The remaining TRP, together with liver degradation products, transported to the brain, heart and skeletal muscle.

Gut microbiota play important roles in absorbing TRP and produce indoles, which act as hydroxyradical scavengers, neuroprotectants, and human AhR (aryl hydrocarbon receptor) selective agonists attenuating inflammations [16]. 
Table 4. Comparison of ratios of tryptophan metabolites per tryptophan. (a) Results of two way ANOVA tests for monopolar major depressive patients; (b) Results of two way ANOVA tests for bipolar depressive patients.

(a)

\begin{tabular}{|c|c|c|c|c|c|}
\hline \multirow{2}{*}{$\begin{array}{c}\text { Diagnosis } \\
\text { Sample name }\end{array}$} & \multicolumn{4}{|c|}{$\begin{array}{l}\text { MDD } \\
\mathrm{n}=38\end{array}$} & \multirow{2}{*}{$\begin{array}{l}\text { significant } \\
\text { differences }\end{array}$} \\
\hline & $\begin{array}{c}\text { young men } \\
\mathrm{n}=7 \\
\mathrm{a}\end{array}$ & $\begin{array}{c}\text { young women } \\
\mathrm{n}=18 \\
\mathrm{~b}\end{array}$ & $\begin{array}{c}\text { old men } \\
\mathrm{n}=3 \\
\mathrm{c}\end{array}$ & $\begin{array}{l}\text { old women } \\
\mathrm{n}=10 \\
\mathrm{~d}\end{array}$ & \\
\hline 5-HTRP/TRP & $0.18 \pm 0.10$ & $0.16 \pm 0.05$ & 0.02 & $0.15 \pm 0.13$ & - \\
\hline serotonin/TRP & $5.24 \pm 5.24$ & $4.47 \pm 3.24$ & $2.02 \pm 1.58$ & $3.19 \pm 3.06$ & - \\
\hline 5-HIAA/TRP & $0.48 \pm 0.46$ & $0.65 \pm 0.64$ & $0.38 \pm 0.46$ & $0.92 \pm 0.91$ & - \\
\hline KYN/TRP & $42.19 \pm 23.68$ & $28.66 \pm 8.23$ & $17.06 \pm 6.65$ & $33.65 \pm 12.12$ & - \\
\hline
\end{tabular}

(b)

\begin{tabular}{|c|c|c|c|c|c|}
\hline \multirow{2}{*}{$\begin{array}{c}\text { Diagnosis } \\
\text { Sample name }\end{array}$} & \multicolumn{4}{|c|}{$\begin{array}{c}\mathrm{BD} \\
\mathrm{n}=17\end{array}$} & \multirow{2}{*}{$\begin{array}{l}\text { significant } \\
\text { differences }\end{array}$} \\
\hline & $\begin{array}{c}\text { young men } \\
\mathrm{n}=2 \\
\mathrm{a}\end{array}$ & $\begin{array}{c}\text { young women } \\
\mathrm{n}=10 \\
\mathrm{~b}\end{array}$ & $\begin{array}{c}\text { old men } \\
\mathrm{n}=3 \\
\mathrm{c}\end{array}$ & $\begin{array}{c}\text { old women } \\
\mathrm{n}=2 \\
\mathrm{~d}\end{array}$ & \\
\hline 5-HTRP/TRP & - & $0.16 \pm 0.04$ & 0.02 & - & - \\
\hline serotonin/TRP & $5.25 \pm 0.97$ & $7.52 \pm 6.48$ & $3.15 \pm 2.07$ & $5.80 \pm 4.97$ & - \\
\hline 5-HIAA/TRP & $0.18 \pm 0.10$ & $0.32 \pm 0.30$ & $0.38 \pm 0.07$ & $3.19 \pm 1.66$ & - \\
\hline KYN/TRP & $19.45 \pm 3.47$ & $23.94 \pm 8.74$ & $31.27 \pm 8.72$ & $23.87 \pm 5.32$ & - \\
\hline
\end{tabular}

Table 5. Comparison of tryptophan metabolites between MMD and BD.

\begin{tabular}{|c|c|c|c|c|c|c|c|c|c|}
\hline \multirow{2}{*}{$\begin{array}{c}\text { Diagnosis } \\
\text { Sample name }\end{array}$} & \multicolumn{4}{|c|}{$\begin{array}{l}\text { MDD } \\
\mathrm{n}=38\end{array}$} & \multicolumn{4}{|c|}{$\begin{array}{c}\text { BD } \\
\mathrm{n}=17\end{array}$} & \multirow[b]{2}{*}{$\begin{array}{l}\text { significant } \\
\text { differences }\end{array}$} \\
\hline & $\begin{array}{l}\text { young men } \\
\qquad \begin{array}{c}\mathrm{n}=7 \\
\mathrm{a}\end{array}\end{array}$ & $\begin{array}{c}\text { young } \\
\text { women } \\
\mathrm{n}=18 \\
\mathrm{~b}\end{array}$ & $\begin{array}{c}\text { old men } \\
\mathrm{n}=3 \\
\mathrm{c}\end{array}$ & $\begin{array}{c}\text { old women } \\
\mathrm{n}=10 \\
\mathrm{~d}\end{array}$ & $\begin{array}{c}\text { young men } \\
\mathrm{n}=2 \\
\mathrm{a}\end{array}$ & $\begin{array}{c}\text { young } \\
\text { women } \\
\mathrm{n}=10 \\
\mathrm{~b}\end{array}$ & $\begin{array}{c}\text { old men } \\
\mathrm{n}=3 \\
\mathrm{c}\end{array}$ & $\begin{array}{c}\text { old women } \\
\mathrm{n}=2 \\
\mathrm{~d}\end{array}$ & \\
\hline 5-HTRP/TRP & $0.18 \pm 0.10$ & $0.16 \pm 0.05$ & 0.02 & $0.15 \pm 0.13$ & - & $0.16 \pm 0.04$ & 0.02 & - & - \\
\hline serotonin/TRP & $5.24 \pm 5.24$ & $4.47 \pm 3.24$ & $2.02 \pm 1.58$ & $3.19 \pm 3.06$ & $5.25 \pm 0.97$ & $7.52 \pm 6.48$ & $3.15 \pm 2.07$ & $5.80 \pm 4.97$ & - \\
\hline 5-HIAA/TRP & $0.48 \pm 0.46$ & $0.65 \pm 0.64$ & $0.38 \pm 0.46$ & $0.92 \pm 0.91$ & $0.18 \pm 0.10$ & $0.32 \pm 0.30$ & $0.38 \pm 0.07$ & $3.19 \pm 1.66$ & * \\
\hline KYN/TRP & $42.19 \pm 23.68$ & $28.66 \pm 8.23$ & $17.06 \pm 6.65$ & $33.65 \pm 12.12$ & $19.45 \pm 3.47$ & $23.94 \pm 8.74$ & $31.27 \pm 8.72$ & $23.87 \pm 5.32$ & - \\
\hline
\end{tabular}

The results of two-way ANOVA were as follows: ${ }^{\star} 5$-HIAA/TRP, Diagnosis $\times$ Sample name interaction: $\mathrm{F}(3,47)=7.409, p<0.01$.

Since we have not done any research about roles of gut microbiota in TRP metabolism, I do not further comment on this issue.

For many people TRP is best known for a substance to generate 5-HT and melatonin [17]. Our results will be shown and discussed later. Since 5-HT is very important in the regulation of mood, anxiety and cognition, people tend to underestimate roles of KYN as a metabolite of TRP. In mammals, the majority of free TRP is degraded through KYN pathway and many metabolites are generated in inflammation, immune responses and neurotransmission [18]. Metabolites 
Table6. Comparison of tryptophan metabolites between MDD and BD.

\begin{tabular}{|c|c|c|c|c|c|}
\hline & $\begin{array}{l}\text { Patients took } \\
\text { SSRI or SNRI } \\
\quad \mathrm{n}=16\end{array}$ & $\begin{array}{c}\text { Patients don't took } \\
\text { SSRI or SNRI } \\
n=39\end{array}$ & Mann-Whitney U & $\mathrm{z}$ & $\mathrm{p}$ \\
\hline Age (years) & $45.75 \pm 13.27$ & $45.28 \pm 11.44$ & 290.500 & -0.399 & 0.690 \\
\hline Hight (m) & $162.08 \pm 7.84$ & $161.42 \pm 7.35$ & 263.000 & -0.909 & 0.363 \\
\hline Weight (kg) & $60.76 \pm 8.08$ & $60.75 \pm 11.91$ & 250.000 & -0.556 & 0.579 \\
\hline BMI & $23.28 \pm 3.76$ & $23.22 \pm 3.44$ & 268.000 & -0.192 & 0.848 \\
\hline CGI-S & $3.88 \pm 0.81$ & $3.90 \pm 0.79$ & 304.500 & -0.158 & 0.875 \\
\hline SDS & $51.93 \pm 12.62$ & $51.53 \pm 10.40$ & 243.000 & -0.261 & 0.794 \\
\hline HDR-S & $18.94 \pm 5.32$ & $17.92 \pm 5.70$ & 268.500 & -0.808 & 0.419 \\
\hline Triptophan $(\mu \mathrm{M})$ & $56.98 \pm 13.00$ & $59.65 \pm 13.72$ & 298.000 & -0.259 & 0.795 \\
\hline L-5-Hydroxytryptophan (nM) & $8.13 \pm 5.08$ & $8.60 \pm 4.27$ & 68.500 & -0.198 & 0.843 \\
\hline Serotonin (nM) & $215.13 \pm 249.99$ & $311.18 \pm 275.84$ & 112.000 & -1.246 & 0.213 \\
\hline Kynurenine (nM) & $1856.63 \pm 596.73$ & $1603.71 \pm 727.67$ & 226.500 & -1.584 & 0.113 \\
\hline 5-Hydroxytryptophol (nM) & $0.00 \pm 0.00$ & $12.60 \pm 11.60$ & - & - & - \\
\hline Tryptophol (nM) & $24.73 \pm 12.85$ & $2.42 \pm 3.57$ & 0.000 & -2.236 & $<0.05$ \\
\hline 5-Hydroxyindoleacetic acid (nM) & $39.05 \pm 47.94$ & $32.60 \pm 31.35$ & 272.000 & -0.741 & 0.459 \\
\hline Indole-3-acetic acid (nM) & $2212.73 \pm 1454.54$ & $1736.09 \pm 1275.02$ & 234.000 & -1.445 & 0.148 \\
\hline Anthranilic acid (nM) & $15.84 \pm 22.44$ & $9.60 \pm 7.95$ & 14.000 & -0.183 & 0.855 \\
\hline Kynurenic acid (nM) & $39.45 \pm 18.07$ & $44.08 \pm 35.54$ & 284.000 & -0.519 & 0.604 \\
\hline Quinaldic acid (nM) & $9.42 \pm 5.76$ & $6.85 \pm 3.70$ & 64.500 & -0.935 & 0.350 \\
\hline 3-Indolebutyric acid (nM) & $19.27 \pm 10.72$ & $17.81 \pm 17.16$ & 216.000 & -1.428 & 0.153 \\
\hline 3-Hydroxykynurenine (nM) & $19.90 \pm 11.97$ & $22.05 \pm 12.75$ & 272.000 & -0.741 & 0.459 \\
\hline 3-hydroxyanthranilic acid (nM) & $24.45 \pm 16.26$ & $24.96 \pm 18.99$ & 183.000 & -0.512 & 0.609 \\
\hline Xanthurenic acid (nM) & $13.05 \pm 5.77$ & $14.52 \pm 11.25$ & 271.000 & -0.276 & 0.782 \\
\hline Melatonin (nM) & $20.65 \pm 31.35$ & $5.38 \pm 8.91$ & 5.000 & -0.866 & 0.386 \\
\hline Quinolinic acid (positive) (nM) & $379.50 \pm 270.30$ & $376.81 \pm 531.39$ & 246.000 & -0.969 & 0.333 \\
\hline 5-HTRP/TRP & $0.13 \pm 0.08$ & $0.16 \pm 0.09$ & 55.000 & -0.962 & 0.336 \\
\hline serotonin/TRP & $3.70 \pm 4.19$ & $5.22 \pm 4.39$ & 110.000 & -1.302 & 0.193 \\
\hline 5-HIAA/TRP & $0.70 \pm 0.78$ & $0.65 \pm 0.84$ & 255.000 & -1.056 & 0.291 \\
\hline KYN/TRP & $33.57 \pm 11.58$ & $27.73 \pm 13.35$ & 205.000 & -1.983 & $<0.05$ \\
\hline
\end{tabular}

Mann-Whitney U test was used for evaluating statistical significance. KYN/TRP ratio was higher in patients who took SSRI/SNRI $(p<0.05)$.

of KYN pathway have been linked to several psychiatric and mental health disorders such as depression and schizophrenia [19]. Sixty percent of KYN are transported from the periphery through blood brain barrier (BBB) and the rest is produced locally. Stress increases many TRP metabolites [20] but these metabolites have antagonizing actions. QA is an N-methyl-p-aspartate receptor agonist and KNA is an NMDAR and $\alpha 7$ nicotinic acetylcholine receptor ( $\alpha 7 \mathrm{nAChR})$ an- 
tagonist. The balance of the levels of these agonist and antagonist is shown to be dysregulated in depression and schizophrenia [21]

Although the concentration of 5-HT has been considered to be low in depressive patients [22], 5-HT concentration in the brains of suicide victims is not low [4]. Therefore, it is not known if 5-HT concentration is decreased in the brains of depressive patients.

By using an ultra high-speed LC/MS, we wanted to know changes in plasma levels of TRP metabolites, especially 5-HT and 5-HIAA (6). Although plasma serotonin levels and 5-HT/TRP ratio were low in depressive patients, the levels of 5-HIAA/TRP were not lower in depressive patients. This result indicates that 5-HT is degraded to 5-HIAA in depressive patients almost to the same extent to healthy old and young women.

We measured the levels of KYN in healthy old men and young women, and depressive patients. TRP seems to be degraded to KYN pathways to the same extent in these three groups.

These results suggest that in depressive patients 5-HT was quickly degraded to 5-HIAA, and this seems to be a reason of low 5-HT levels in depressive patients.

Recently we found that there are significant gender and age differences in plasma levels of TRP metabolites [23]. Plasma levels of 5-HT, 5-HIAA, KYN, AA are higher in young women and old men that young men and old women, but plasma levels of 3-HKYN and XA are higher in young men and old women than young women and old men.

These results mean that there are significant age and gender difference in plasma levels of TRP metabolites.

We then measured plasma levels of TRP metabolites in MMD and BD and found that such significant gender and age difference disappear in MMD patients. Although some changes exist in BD patients, they are not so significant as shown in healthy people.

It is not probable that such changes are due to use of drugs for depression.

Probably in patients of depression severe hormonal change may influence TRP metabolism.

As to a relationship between serotonin pathway and KYN pathway, Lapin IP suggested that in depression tryptophan 2,3-dixygenase in the liver shunted metabolism of serotonin away from 5-HT production to KYN production, resulting in serotonin deficiency [24]. KYN, QN and 3-HKN were shown to be anxiogenic and KNYA were anxiolytic [25]. From these results, he tried to explain the effects of anti-depressive drugs.

Our results do not support this hypothesis. Metabolites of KYN pathways were not high in depressive patients.

There have not been enough studies as to 5-HT levels in the brain of patients of bipolar depression. Serotonin levels in cerebrospinal fluids of patients of bipolar depression were shown to be high [26] or normal [27]. So, it seems to be very important to discriminate monopolar and bipolar depression to study roles of serotonin in the pathogenesis of disease. 
Our results show that plasma 5-HT levels were low and metabolites of KYN pathway were not changed in patients of monopolar depression.

Comparison of TRP metabolites in healthy people and MMD or BD patients will be reported soon.

Finally, relationship of TRP metabolites levels between brain and plasma should be discussed.

It is well known that 5-HT does not pass the blood brain barrier [28]. It has been suggested that the integrity of the endothelial cell lining of the cerebrovascular bed constitutes a morphological blood-brain barrier mechanism to neurotransmitter monoamines. Circulating monoamines are prevented from entering the brain primarily at the luminal membrane of the endothelial ling.

So, we wonder whether the measurements of TRP levels in the blood may represent their changes in the brain.

We have reported changes in TRP metabolites levels in the bran and plasma after the application of electric shocks in rats [29] [30] [31] [32]. Most of TRP metabolites including 5-HT and 5-HIAA in the brain and plasma increased immediately after electric shock and returned to normal $30 \mathrm{~min}$. later. These results suggest that plasma levels of TRP metabolites may represent those in the brain, of which the reasons we could not speculate. However, we can get some metabolic informations in the brain by analyzing plasma levels of TRP metabolites such as 5-HT, 5-HIAA, or KYN.

\section{Ethics}

This work has been approved by Institutional Review Board of Showa University Northern Yokohama Hospital (No. 1508-11) and has been carried out in accordance with The Code of Ethics of the World Medical Association (Declaration of Helsinki) for experiments.

\section{Financial Support}

The present study was financially supported by grants in-aid from the Ito Memorial Foundation and NPO "International products on Food and Health".

\section{Acknowledgements}

Experiments were designed and performed by all of the authors. AT wrote a manuscript. Statistical analyses were done by MH. All authors read the manuscript and approve the final manuscript. All the authors had responsibilities for a final content. There is no conflict of interest for any author.

\section{Conflicts of Interest}

The authors declare no conflicts of interest regarding the publication of this paper.

\section{References}

[1] Rhodes, K.M., Turner, R.M. and Higgins, J.P. (2015) Predictive Distributions Were 
Developed for the Extent of Heterogeneity in Meta-Analyses of Continuous Outcome Data. Journal of Clinical Epidemiology, 68, 52-60.

https://doi.org/10.1016/j.jclinepi.2014.08.012

[2] GBD 2015 DALYs and HALE Collaborators (2016) Global, Regional, and National Disability-Adjusted Life-Years (DALYs) for 315 Diseases and Injuries and Healthy Life Expectancy (HALE), 1990-2015: A Systematic Analysis for the Global Burden of Disease Study 2015. The Lancet, 388, 1603-1658. https://doi.org/10.1016/S0140-6736(16)31460-X

[3] Cipriani, A., Furukawa, T.A., Salanti, G., Chaimani, A., Atkinson, L.Z., Ogawa, Y., Leucht, S., Ruhe, H.G., Turner, E.H., Higgins, J.P.T., Egger, M., Takeshima, N., Hayasaka, Y., Imai, H., Shinohara, K., Tajika, A., Ioannidis, J.P.A. and Geddes, J.R. (2018) Comparative Efficacy and Acceptability of 21 Antidepressant Drugs for the Acute Treatment of Adults with Major Depressive Disorder: A Systematic Review and Network Meta-Analysis. The Lancet, 391, 1357-1366.

https://doi.org/10.1016/S0140-6736(17)32802-7

[4] Oquendo, M.A., Sullivan, G.M., Sudol, K., Baca-Garcia, E., Stanley, B.H., et al. (2014) Toward a Biosignature for Suicide. American Journal of Psychiatry, 171, 1259-1277. https://doi.org/10.1176/appi.ajp.2014.14020194

[5] Matsuoka, K., Kato, K., Takao, T., Ogawa, M., Ishii, Y., Shimizu, F., Masuda, J. and Takada, A. (2016) Concentrations of Various Tryptophan Metabolites Increase in Patients of Diabetes Mellitus Compared to Healthy Aged Male Adults. Diabetology International, 8, 69-72. https://doi.org/10.1007/s13340-016-0282-y

[6] Takada, A., Shimizu, F. and Masuda, J. (2018) Measurement of Plasma Tryptophan Metabolites: Clinical and Experimental Application for Depression and Stress States Assessment. In: Dragoi, C.M., Ed., Melatonin-Molecular Biology, Clinical and Pharmaceutical Approaches, Springer, New York. https://doi.org/10.5772/intechopen.78560

[7] Shimizu, F., Ishii, Y., Ogawa, M., Takao, T., Matsuoka, K., Kato, K. and Takada, A. (2018) Plasma Levels of Tryptophan Metabolites in Healthy Young and Old Men and Women, and Patients of Type 2 Diabetes Mellitus (T2DM). Obesity: Open Access, 4, 138. https://doi.org/10.16966/2380-5528.138

[8] Takada, A., Shimizu, F. and Masuda, J. (2019) Plasma Levels of Tryptophan Metabolites in Patients of Type 2 Diabetes Mellitus. In: Watson, R. and Preedy, V., Eds., Bioactive Food as Dietary Interventions for Diabetes, 2nd Edition, Academic Press, New York, 265-276. https://doi.org/10.1016/B978-0-12-813822-9.00017-5

[9] American Psychiatric Association (2013) Diagnostic and Statistical Manual of Mental Disorders. 5th Edition (DSM-5), APA, Washington DC. https://doi.org/10.1176/appi.books.9780890425596

[10] Guy, W. (1976) Clinical Global Impressions ECDEU Assessment Manual for Psychopharmacology, Revised (DHEW Publ. No. ADM 76-338). National Institute of Mental Health, Rockville, MD, 218-222. https://doi.org/10.1037/e591322011-001

[11] Zung, W.W.K. (1965) A Self-Rating Depression Scale. Archives of General Psychiatry, 12, 63-70. https://doi.org/10.1001/archpsyc.1965.01720310065008

[12] Hamilton, M. (1960) A Rating Scale for Depression. The Journal of Neurology, Neurosurgery, and Psychiatry, 23, 56-62. https://doi.org/10.1136/jnnp.23.1.56

[13] Takada, A., Shimizu, F., Takao, T. and Masuda, J. (2018) Measurement of Tryptophan Metabolites in Healthy Old Men and Patients of Type 2 Diabetes Mellitus (T2DM). Food and Nutrition Sciences, 9, 1206-1220. https://doi.org/10.4236/fns.2018.910087 
[14] Cervenka, I., Agudelo, L.Z. and Ruas, J.L. (2017) Kynurenines: Tryptophan's Metabolites in Exercise, Inflammation, and Mental Health. Science, 357, eaaf9794. https://doi.org/10.1126/science.aaf9794

[15] NR Council (1989) Recommended Dietary Allowances. 10th Edition, The National Academies Press, Washington DC.

[16] Zelante, T., Iannitti, R.G., Cunha, C., De Luca, A., Giovannini, G., Pieraccini, G.L., et al. (2013) Tryptophan Catabolites from Microbiota Engage Aryl Hydrocarbon Receptor and Balance Mucosal Reactivity via Interleukin-22. Immunity, 39, 372-385. https://doi.org/10.1016/j.immuni.2013.08.003

[17] Takada, A. and Curzon, G. (1995) Serotonin in the Central Nervous System and Periphery. International Congress Series 1088, Excerpta Medica.

[18] Stone, T.W., Stoy, N. and Darlington, L.G. (2013) An Expanding Range of Targets for Kynurenine Metabolites of Tryptophan. Trends in Pharmacological Sciences, 34, 136-143. https://doi.org/10.1016/j.tips.2012.09.006

[19] Chen, Y. and Guillemin, G.J. (2009) Kynurenine Pathway Metabolites in Humans: Disease and Healthy States. International Journal of Tryptophan Research, 2, 1-19. https://doi.org/10.4137/IJTR.S2097

[20] Pawlak, D., Takada, Y., Urano, T. and Takada, A. (2000) Serotonergic and Kynurenic Pathways in Rats Exposed to Foot Shock. Brain Research Bulletin, 52, 197-206. https://doi.org/10.1016/S0361-9230(00)00252-5

[21] Müller, N. and Schwarz, M.J. (2007) The Immune-Mediated Alteration of Serotonin and Glutamate: Towards an Integrated View of Depression. Molecular Psychiatry, 12, 988-1000. https://doi.org/10.1038/sj.mp.4002006

[22] Mann, J.J., Arango, V., Marzuk, P.M., Theccanat, S. and Reis, D.J. (1989) Evidence for the 5-HT Hypothesis of Suicide. A Review of Postmortem Studies. The British Journal of Psychiatry, 155, 7-14. https://doi.org/10.1192/S000712500029171X

[23] Masuda, J., Karayama, M., Suda, T., Maekawa, M., Shimizu, F. and Takada, A. (2019) Effects of Gender, Age, and Clot Formation on the Measurements of Tryptophan Metabolites in Blood. Food and Nutrition Sciences, 10, 761-775. https://doi.org/10.4236/fns.2019.107056

[24] Lapin, I.P. (1973) Kynurenines as Probable Participants of Depression. Pharmakopsychiatrie, Neuro-Psychopharmakologie, 6, 273-279.

https://doi.org/10.1055/s-0028-1094391

[25] Lapin, I.P. (1998) Antagonism of Kynurenic Acid to Anxiogens in Mice. Life Sciences, 63, 231-236. https://doi.org/10.1016/S0024-3205(98)00404-4

[26] Pålsson, E., Sellgren, C., Rydén, E., Kizza, R., Pelanis, A., Zetterberg, H., Blennow, K. and Landén, M. (2017) Cerebrospinal Fluid Monoamine Metabolite Profiles in Bipolar Disorder, ADHD, and Controls. Journal of Neural Transmission, 124 1135-1143. https://doi.org/10.1007/s00702-017-1746-3

[27] Berrettini, W.H., Nurnberger Jr., J.I., Scheinin, M., Seppala, T., Linnoila, M., Narrow, W., Simmons-Alling, S. and Gershon, E.S. (1985) Cerebrospinal Fluid and Plasma Monoamines and Their Metabolites in Euthymic Bipolar Patients. Biological Psychiatry, 20, 257-269. https://doi.org/10.1016/0006-3223(85)90055-1

[28] Hardebo, J.E. and Owman, C. (1980) Barrier Mechanisms for Neurotransmitter Monoamines and Their Precursors at the Blood-Brain Interface. Annals of Neurology, 1, 1-31. https://doi.org/10.1002/ana.410080102

[29] Malyszko, J., Urano, T., Takada, Y. and Takada, A. (1995) Amino Acids, Serotonin, and 5-Hydroxyindole Acetic Acid Following Foot Shock to Rats. Brain Research, 
36, 137-140. https://doi.org/10.1016/0361-9230(94)00178-4

[30] Malyszko, J., Urano, T., Yan, D., Serizawa, Y., Koima, Y., Takada, Y. and Takada, A. (1994) Foot Shock Induced Changes Blood and Brain Serotonin and Related Substances in Rats. The Japanese Journal of Physiology, 44, 35-47. https://doi.org/10.2170/jiphysiol.44.35

[31] Pawlak, R., Takada, Y., Takahashi, H., Urano, T., Ihara, H., Nagai, N. and Takada, A. (2000) Differential Effects of Nicotine against Stress Induced Changes in Dopaminergic System in Rat Striatum and Hippocampus. European Journal of Pharmacology, 387, 171-177. https://doi.org/10.1016/S0014-2999(99)00778-5

[32] Pawlak, D., Takada, Y., Urano, T. and Takada, A. (2000) Serotonergic and Kynurenic Pathways in Rats Exposed to Foot Shock. Brain Research Bulletin, 52, 197-205. https://doi.org/10.1016/S0361-9230(00)00252-5

\section{Abbreviation}

tryptophan (TRP), serotonin (5-HT), 5-hydroxyindole acetic acid (5-HIAA), indole-3-acetic acid (IAA), kynurenine (KYN), xanthurenic acid (XA), anthranilic acid (AA), kynurenic acid (KNA), 3-hydroxykynurenine (3-HKN), indoleamine dioxygenase (IDO), tryptophan dioxygenase (TDO). Selective serotonin uptake inhibitor (SSRI), serotonin epinephrine reuptake inhibitor (SNRI), clinical global impression-severity scale (CGI-s), self-rating depression scale (SDS), Hamilton depression rating scale(HDRS). 